\title{
Risk factors of atrial fibrillation recurrence despite successful radiofrequency ablation of accessory pathway: At 11 years of follow-up
}

Michał Orczykowski ${ }^{1}$, Piotr Urbanek ${ }^{1}$, Robert Bodalski ${ }^{1}$, Paweł Derejko ${ }^{2}$, Grzegorz Warmiński ${ }^{1}$, Małgorzata Łodyga ${ }^{1}$, Damian Łasocha ${ }^{1}$, Łukasz Mazurkiewicz ${ }^{3}$, Maciej Dąbrowski ${ }^{4}$, Paweł Tyczyński ${ }^{4}$, Joanna Zakrzewska-Koperska ${ }^{1}$, Rafał Baranowski ${ }^{1}$, Artur Oręziak ${ }^{1}$, Maciej Sterliński ${ }^{1}$, Maria Bilińska ${ }^{1}$, Łukasz Szumowski ${ }^{1}$

${ }^{1}$ Arrhythmia Department, Institute of Cardiology, Warsaw, Poland

${ }^{2}$ Cardiology and Internal Medicine Department, Medicover Hospital, Warsaw, Poland

${ }^{3}$ Department of Cardiomyopathy, Institute of Cardiology, Warsaw, Poland

${ }^{4}$ Department of Interventional Cardiology and Angiology, Institute of Cardiology, Warsaw, Poland

\begin{abstract}
Background: Previous reports on patients with radiofrequency catheter ablation (RFCA) of accessory pathway $(A P)$ and atrial fibrillation $(A F)$ include only short follow-up periods. The aim of this study was to analyze predictors of recurrence of AF in patients after successful RFCA of APs over long term follow-up periods.

Methods: Of the 1,007 patients who underwent non-pharmacological treatment of APs (between the years 1993-2008), data of 100 consecutive patients were retrospectively analyzed (75 men, mean age $43.6 \pm 14.7$ ), with the longest period of follow-up (mean $11.3 \pm 3.5$ years) after successful RFCA of AP. In Group 1, there were 72 patients (54 men, mean age $40.66 \pm 13.85$ years) without documented episodes of AF after RFCA of AP. Group 2 consisted of 28 patients (21 men, mean age $50.79 \pm 14.49$ years) with $A F$ episodes despite successful elimination of $A P$.

Results: In univariate analysis, patients from Group 1 were significantly younger at the time of ablation than patients from Group 2 (40.66 \pm 13.85 vs. $50.79 \pm 14.49$ years; $p=0.002)$, had shorter history of $A F$ episodes $(4.11 \pm 4.07 v$ s. $8.25 \pm 7.50$ years; $p=0.024)$ and had less frequently documented atrial tachycardia (AT) prior to ablation (3.39 vs. $20.00 \%$; $p=0.022$ ). In multivariate analysis, the history of $A F$ in years ( $p=0.043)$, was an independent risk factor for $A F$ recurrences.

Conclusions: Older patient age, longer history of AF and AT prior to RFCA of APs identified a subgroup of patients who required additional treatment. In the multivariate analysis, the history of $A F$ in years ( $p=0.043$ ) was a risk factor for AF recurrence. (Cardiol J 2017; 24, 6: 597-603)

Key words: Wolff-Parkinson-White syndrome, atrial fibrillation, radiofrequency ablation, accessory pathway, follow-up
\end{abstract}

\section{Introduction}

Pre-excitation occurs in $0.1-0.3 \%$ of the general population [1-5]. Among patients with accessory pathways (APs), the incidence of atrial fibrillation (AF) has been reported to be as high as $30 \%$, which is far greater than the incidence of $\mathrm{AF}$ in the general population where it is $3-4 \%[6,7]$. Such a high occurrence of AF in patients with APs is still a subject of debate [6-8]. Patients with

Address for correspondence: Michał Orczykowski, MD, PhD, Institute of Cardiology, Arrhythmia Department, ul. Alpejska 42, 04-628 Warszawa, Poland, tel: +48 6967755 33, fax: +48 223434 520, e-mail: morczykowski@gazeta.pl 


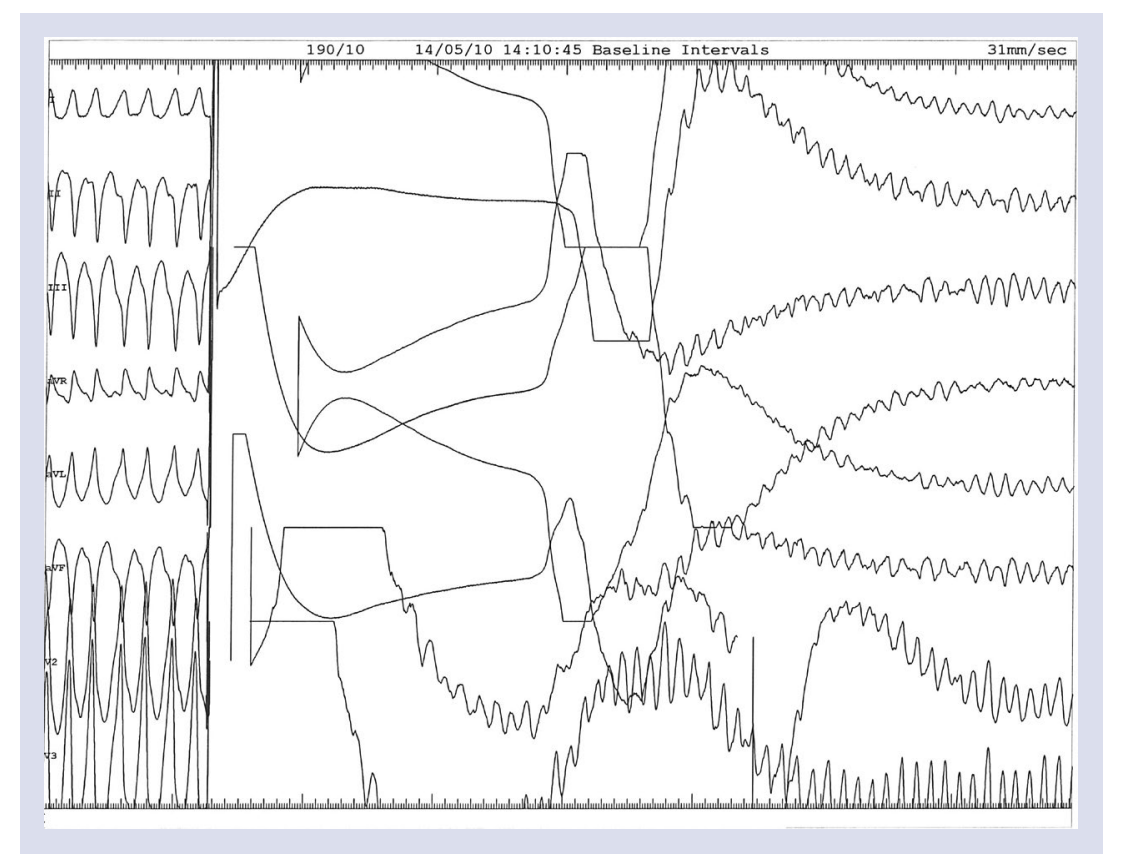

Figure 1. Inadvertently induced ventricular fibrillation by non-synchronized cardioversion of atrial fibrillation conducted by the accessory pathway.

pre-excitation are at risk of sudden cardiac death (SCD). The generally accepted mechanism for SCD in Wolff-Parkinson-White syndrome (WPW) is the fast conduction of $\mathrm{AF}$ to the ventricles over an $\mathrm{AP}$ with subsequent degeneration into ventricular fibrillation (VF) [9-11]. The current guidelines recommend radiofrequency catheter ablation (RFCA) as the first line therapy in patients having AF and WPW [12-14].

Research has demonstrated that following successful surgical dissection of APs, there is an almost complete elimination of paroxysmal AF; however, the patients in these studies were young [15-17]. Less information exists concerning RFCA due to the enrollment of small groups of patients in previous studies and a short follow-up period [18-20].

Among patients with WPW and AF, ablation of AP may have a sufficient clinical impact of preventing recurrence of AF in selected patients [21]. Some patients need additional attention and treatment [19].

The aim of this study was to analyze the predictors of recurrence of AF in patients after successful RFCA of AP during long term follow-up and ascertain the impact of ablation on future AF risk $[18,19]$.

\section{Methods}

\section{Study patients}

Of the 1,007 patients (mean age $35.00 \pm 15$; $45 \%$ female) who underwent non-pharmacological treatment of APs (years 1993-2008), 281 patients (28\%) had AF (Fig. 1).

Data were retrospectively analyzed for 100 consecutive patients ( 75 male, 25 female, mean age $43.6 \pm 14.7$ years, range $15-74$ ), with the longest period of follow-up (mean $11.3 \pm 3.5$ years) after successful RFCA of AP in order to accomplish the longest follow-up ever published on a large group of patients. This was a retrospective analysis, nevertheless the follow-up was prospectively designed.

Patients with persistent AF prior to ablation were excluded and patients with a history of VF were the topic of a previous publication [22].

Clinical, electrocardiographic, electrophysiological, and echocardiographic data were analyzed.

\section{Ethics}

The study was conducted according to the Declaration of Helsinki. All patients provided written, informed consent for the ablation procedure and for the scientific data analysis. The consent for the procedure was approved by the Institutional Ethics Committee.

\section{Electrophysiological study}

In patients with a history of AF, the aim of the electrophysiological study was to confirm the diagnosis and to perform the ablation procedure as safely as possible. Aggressive atrial stimula- 


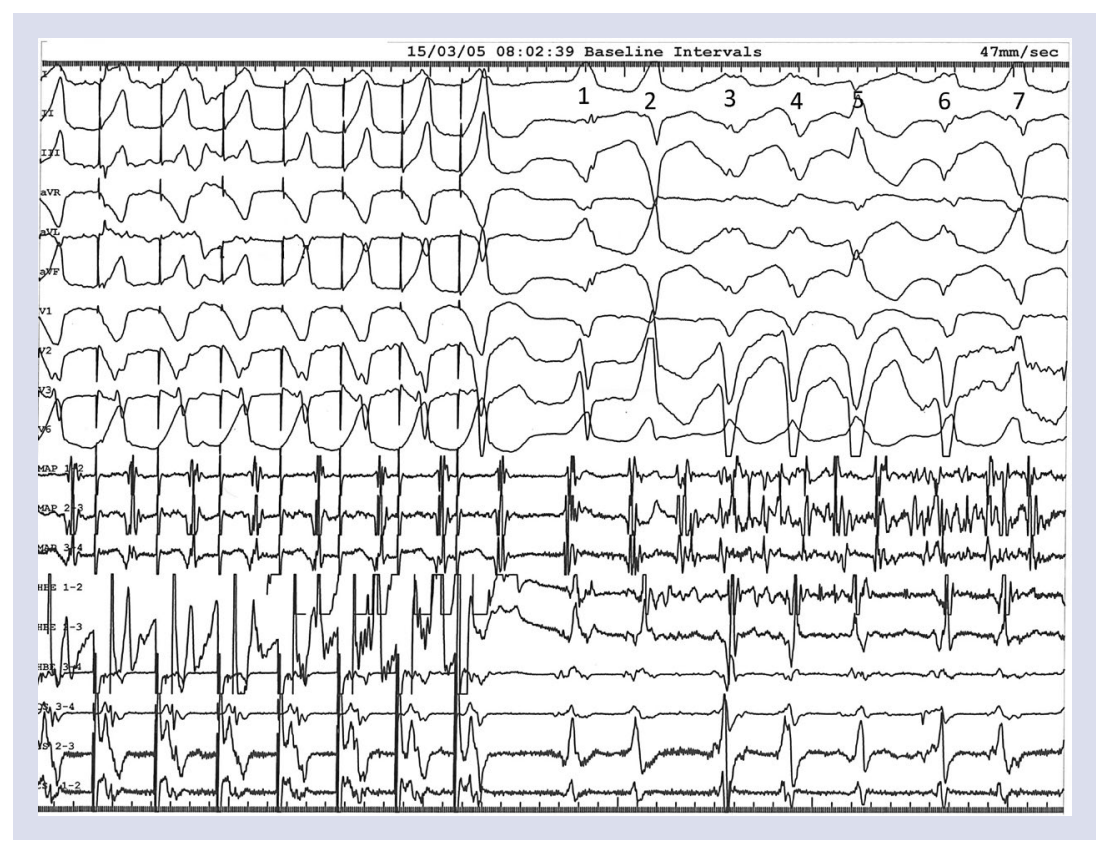

Figure 2. Atrial fibrillation with changing pre-excitation induced by pacing from right ventricle in patient with history of ventricular fibrillation episode, multiple accessory pathways and Ebstein anomaly. Following evolutions: QRS 2, 7 — inferoparaseptal pathway (QRS $150 \mathrm{~ms}$ ), QRS 3, 4, 6 — right antero-superior pathway (QRS $190 \mathrm{~ms}$ ).

tion was therefore avoided in order to reduce the risk of inducing life-threatening arrhythmia. As amiodarone was administered prior to admission to this institution in most patients after a $\mathrm{VF}$ or $\mathrm{AF}$ episode with fast ventricular response, the refractory periods of $\mathrm{AP}$ and $\mathrm{AV}$ node were not analyzed.

\section{Statistical analysis}

Continuous variables were expressed as means and standard deviations (SD). The Yates-corrected $\chi^{2}$ test was used to compare fractions of two categorical variables and the maximum-likelihood $\chi^{2}$ test was used when larger frequency tables were constructed. The differences in the means of continuous variables were investigated with t-test and analysis of variance (ANOVA). Post-hoc comparisons in the analysis of variance were made with Tukey's range test. The Pearson correlation was used to investigate relationships between two continuous variables. $\mathrm{P}<0.05$ was considered statistically significant.

\section{Definitions}

The location of the AP was retrospectively described using the anatomic nomenclature proposed by the European Society of Cardiology and the North American Society of Pacing and Electrophysiology [23].

Accessory pathways were grouped into three locations: left free wall (left superior, left poste- riorsuperior, left posterior, left posteroinferior and left inferior), septal-paraseptal region (superoparaseptal, septal and inferoparaseptal), and right free wall (right superior, right superoanterior, right anterior, right anteroinferior and right inferior) [24].

Multiple APs (or an AP with multiple ventricular or atrial insertions, Fig. 2) was defined as previously described [25].

\section{Follow-up}

Patients underwent routine follow-up at 6 and 12 months after ablation in the outpatient clinic of the hospital or by the referring physician. Echocardiography was performed on all patients and they had undergone two or more Holter electrocardiogram (ECG) recordings. After 12 months, patients with no palpitation and no pre-excitation in ECG were referred to regional outpatient cardiovascular centers. There a Holter ECG recordings were performed at least once a year.

Late follow-up after a mean of $11.3 \pm 3.5$ years was performed by questionnaire designed by the authors or by direct telephone interviews with patients and/or via direct contact with the referring physician. During late follow-up, 73\% of patients were still under cardiac supervision in a regional outpatient clinic. When patients had documented arrhythmias during the follow-up period, informa- 
Table 1. Clinical data.

\begin{tabular}{lccc}
\hline & Group 1 & Group 2 & P \\
\hline Age during ablation [years] & $40.66 \pm 13.85$ & $50.79 \pm 14.49$ & 0.002 \\
History of AF [years] & $4.11 \pm 4.07$ & $8.25 \pm 7.50$ & 0.024 \\
History of AVRT [years] & $17.26 \pm 11.85$ & $24.25 \pm 12.55$ & NS \\
Follow-up [years] & $11.24 \pm 3.20$ & $11.82 \pm 4.29$ & NS \\
Age during 1 episode of AF [years] & $37.67 \pm 14.71$ & $40.35 \pm 14.68$ & NS \\
Age during 1 episode of AVRT [years] & $21.81 \pm 13.49$ & $25.64 \pm 18.94$ & NS \\
Male gender [\%] & 75.00 & 75.00 & NS \\
Body mass index & $25.73 \pm 3.41$ & $26.72 \pm 3.32$ & NS \\
Hypertension [\%] & 25.81 & 33.33 & NS \\
Dyslipidemia [\%] & 43.0 & 46.66 & NS \\
Diabetes [\%] & 5.26 & 8.70 & NS \\
Heart failure [\%] & 0.0 & 0.0 & NS \\
\hline
\end{tabular}

AF — atrial fibrillation; AVRT — atrioventricular reentrant tachycardia; NS — not significant

tion regarding these arrhythmias were obtained from the referring physicians and documentation was sought. The methodology of late follow-up contact was published previously [19].

\section{Results}

In Group 1, there were 72 patients (54 males, 18 females; mean age: $40.66 \pm 13.85$ years) without documented episodes of AF after RFCA of AP. Group 2 consisted of 28 patients ( 21 males, 7 females; mean age: $50.79 \pm 14.49$ years) with $\mathrm{AF}$ episodes despite the successful elimination of AP. 119 RFCA of APs in 100 patients were performed (range 1-5, mean 1.2).

\section{Clinical data}

Clinical data are presented in Table 1. Using univariate analysis, patients from Group 1 were found to be significantly younger, at the time of ablation, than were patients from Group 2: $40.66 \pm 13.85$ vs. $50.79 \pm 14.49$ years $(\mathrm{p}=0.002)$, respectively. Furthermore, patients from Group 1 had a shorter history of AF episodes: $4.11 \pm 4.07$ vs. $8.25 \pm 7.50$ years $(\mathrm{p}=0.024)$, respectively.

\section{Electrocardiographic \\ and electrophysiological data}

Data are presented in Table 2. In Group 1, there were significantly less patients with documented atrial tachycardia (AT) prior to ablation 3.39 vs. $20.00(\mathrm{p}=0.022)$, respectively.

\section{Echocardiographic data}

Echocardiographic assessment was performed in all patients from both groups (Table 3).

\section{Deaths during follow-up}

During follow-up, 2 patients died despite successful RFCA of AP. Both had a normal ejection fraction as well as hypertension and dyslipidemia. The first patient died at the age of 69, due to cancer 9 years after RFCA of AP. According to the family, the second patient died at age of 71, 6 months after RFCA, due to respiratory failure.

\section{Multivariate analysis}

Using multivariate analysis, only a history of $\mathrm{AF}$ in years $(\mathrm{p}=0.043)$ were independent risk factors for $\mathrm{AF}$ recurrences.

\section{Discussion}

The present study investigated $\mathrm{AF}$ recurrence in one of the largest cohorts of patients with paroxysmal AF and RFCA of APs. It was sought to describe the long-term outcomes of AP and AF patients and ascertain the impact of ablation on future $\mathrm{AF}$ risk.

Data of patients with AF and RFCA of APs published previously reported a relatively short follow-up that lasted from $23.9 \pm 12$ to $35 \pm 12$ months $[18,19]$. Research herein indicates that the present study has the longest follow-up period of 11 years. This study demonstrates that a shorter 
Table 2. Electrocardiographic and electrophysiological data.

\begin{tabular}{lccc}
\hline & Group 1 & Group 2 & P \\
\hline Documented AT [\%] & 3.39 & 20.00 & 0.022 \\
Overt pre-excitation [\%] & 83.33 & 71.43 & NS \\
Intermittent pre-excitation [\%] & 6.94 & 10.71 & NS \\
Concealed AP [\%] & 9.73 & 17.86 & NS \\
Documented AVRT [\%] & 79.17 & 67.86 & NS \\
Documented AVNRT [\%] & 2.94 & 8.70 & NS \\
Documented AF [\%] & 9.43 & 10.00 & NS \\
Documented VF [\%] & 1.41 & 3.57 & NS \\
Mean SPRR [ms] & $250.00 \pm 67.85$ & $274.62 \pm 100.88$ & NS \\
Mean max. AVRT [bpm] & $220.77 \pm 41.59$ & $208.13 \pm 28.10$ & NS \\
Mean min. AVRT [bpm] & $182.32 \pm 27.13$ & $178.57 \pm 26.85$ & NS \\
RFW [\%] & 12.50 & 21.43 & NS \\
LFW [\%] & 61.12 & 50.00 & NS \\
Midseptal/posteroseptal [\%] & 26.38 & 28.57 & NS \\
MAP [\%] & 22.86 & 25.00 & NS \\
ECV [\%] & 43.18 & 45.45 & NS \\
\hline
\end{tabular}

AF — atrial fibrillation; AP — accessory pathway; AT — atrial tachycardia; AVNRT — atrioventricular nodal reciprocating tachycardia; AVRT - atrioventricular reciprocating tachycardia; ECV — electrical cardioversion; LFW - left free wall; MAP - multiple accessory pathways; NS - not significant; mean SPRR - mean shortest pre-excited RR; RFW — right free wall; VF — ventricular fibrillation

Table 3. Echocardiographic data.

\begin{tabular}{lccc}
\hline & Group 1 & Group 2 & P \\
\hline Ejection fraction [\%] & $66.40 \pm 7.58$ & $65.29 \pm 5.97$ & NS \\
Shortening fraction [\%] & $37.88 \pm 6.41$ & $36.29 \pm 4.69$ & NS \\
LA [cm] & $3.68 \pm 0.55$ & $3.74 \pm 0.57$ & NS \\
LVDD [cm] & $4.97 \pm 0.38$ & $5.03 \pm 0.44$ & NS \\
LVSD [cm] & $3.15 \pm 0.53$ & $3.17 \pm 0.34$ & NS \\
LVEDV [mL] & $115.91 \pm 29.02$ & $121.39 \pm 25.34$ & NS \\
LVESV [mL] & $41.05 \pm 13.99$ & $39.79 \pm 9.62$ & NS \\
Stroke volume [mL] & $76.18 \pm 19.97$ & $80.14 \pm 19.31$ & NS \\
IVSD [cm] & $1.07 \pm 0.13$ & $1.07 \pm 0.15$ & NS \\
Additional heart diseases [\%] & 8.06 & 10.00 & NS \\
\hline
\end{tabular}

IVSD — interventricular septal diameter; LA — left atrium; LVDD — left ventricular diastolic diameter; LVSD — left ventricular systolic diameter; LVEDV — left ventricular end-diastolic volume; LVESV — left ventricular end-systolic volume; NS — not significant

period between the diagnosis of $\mathrm{AF}$ in patients with APs to the treatment and elimination of APs reduces the risk of future $\mathrm{AF}$ episodes. This is also observed in patients without AP [26]. The present research finds that this correlation has not yet been reported for $\mathrm{AP}$ and $\mathrm{AF}$ patients.

Moreover, one of the most important findings of this study is that patients with AF recurrence after the elimination of APs is more commonly documented in AT prior to ablation. Miyamoto et al. [20] reported a correlation of premature atrial complexes prior to ablation of atrioventricular nodal reciprocating tachycardia (AVNRT) or AP and higher recurrences of $\mathrm{AF}$ after a successful procedure.

More frequently documented episodes of AT in patients with $\mathrm{AF}$ after RFCA of AP in these patients, may suggest that, in Group 2, there were more patients with focal $\mathrm{AF}$, triggers in pulmonary veins, or focal re-entry of electrical signal propagation in the left atrium [13]. 
These findings support the observations of Dagres et al. [19], which indicated that age is a risk factor for AF recurrence. Patients with recurrences of AF several years after elimination of AP, probably revealed a second mechanism of AF episodes, which is not connected with AP.

\section{Limitations of the study}

Data were collected on ablation procedures, retrospectively. However, long-term follow-up was prospectively designed. It is not possible to refute the theory that some patients from Group 1 were asymptomatic of or did not report experiencing $\mathrm{AF}$.

\section{Conclusions}

Older patient age, longer history of $\mathrm{AF}$, and the presence of AT episodes prior to RFCA of APs in patients with an AP and AF indicated the need for additional treatment. Upon multivariate analysis, only a history of AF ( $\mathrm{p}=0.043)$ was an independent risk factor for $\mathrm{AF}$ recurrence.

This data may assist physicians to advise patients properly regarding the future risk of arrhythmia as well as anticoagulation.

\section{Acknowledgements}

We would like to thank Editage (www.editage. com) for English language editing.

Funding sources: This research received no grants from any funding agency in the public, commercial, or not-for-profit sectors.

\section{Conflict of interest: None declared}

\section{References}

1. Krahn AD, Manfreda J, Tate RB, et al. The natural history of electrocardiographic preexcitation in men. The Manitoba Follow-up Study. Ann Intern Med. 1992; 116(6): 456-460, doi: 10.7326/0003-4819-116-6-456, indexed in Pubmed: 1739235.

2. Rodriguez LM, Smeets JL, de Chillou C, et al. The 12-lead electrocardiogram in midseptal, anteroseptal, posteroseptal and right free wall accessory pathways. Am J Cardiol. 1993; 72(17): 1274-1280, doi: 10.1016/0002-9149(93)90296-o, indexed in Pubmed: 8256703.

3. Della Bella P, Brugada P, Talajic M, et al. Atrial fibrillation in patients with an accessory pathway: importance of the conduction properties of the accessory pathway. J Am Coll Cardiol. 1991; 17(6): 1352-1356, doi: 10.1016/s0735-1097(10)80146-9, indexed in Pubmed: 2016453.

4. Rodriguez LM, Chillou Cde, Schläpfer J, et al. Age at onset and gender of patients with different types of supraventricular tachycardias. The American Journal of Cardiology. 1992; 70(13): 1213-1215, doi: 10.1016/0002-9149(92)90060-c.
5. Orczykowski M, Derejko P, Bodalski R, et al. Radiofrequency catheter ablation of accessory pathways in patients with Ebstein's anomaly: At 8 years of follow-up. Cardiol J. 2017; 24(1): 1-8, doi: 10.5603/CJ.a2016.0111, indexed in Pubmed: 27910083.

6. Berkman NL, Lamb LE. The Wolff-Parkinson-White Electrocardiogram. N Engl J Med. 1968; 278(9): 492-494, doi: 10.1056/ nejm196802292780906.

7. Miyasaka Y, Barnes ME, Gersh BJ, et al. Secular trends in incidence of atrial fibrillation in Olmsted County, Minnesota, 1980 to 2000 , and implications on the projections for future prevalence. Circulation. 2006; 114(2): 119-125, doi: 10.1161/CIRCULATIONAHA.105.595140, indexed in Pubmed: 16818816.

8. Iesaka Y, Yamane T, Takahashi A, et al. Retrograde multiple and multifiber accessory pathway conduction in the Wolff-Parkinson-White syndrome: potential precipitating factor of atrial fibrillation. J Cardiovasc Electrophysiol. 1998; 9(2): 141-151, doi: 10.1111/j.1540-8167.1998.tb00895.x, indexed in Pubmed: 9511888.

9. Dreifus LS, Haiat R, Watanabe Y, et al. Ventricular fibrillation. A possible mechanism of sudden death in patients and Wolff-Parkinson-White syndrome. Circulation. 1971; 43(4): 520-527, doi: 10.1161/01.cir.43.4.520, indexed in Pubmed: 5573385.

10. Klein GJ, Bashore TM, Sellers TD, et al. Ventricular fibrillation in the Wolff-Parkinson-White syndrome. N Engl J Med. 1979; 301(20): 1080-1085, doi: 10.1056/NEJM197911153012003, indexed in Pubmed: 492252.

11. Basso C, Corrado D, Rossi L, et al. Ventricular preexcitation in children and young adults: atrial myocarditis as a possible trigger of sudden death. Circulation. 2001; 103(2): 269-275, doi: 10.1161/01.cir.103.2.269, indexed in Pubmed: 11208688.

12. Blomström-Lundqvist C, Scheinman MM, Aliot EM, et al. ACC/ /AHA/ESC guidelines for the management of patients with supraventricular arrhythmias: executive summary: a report of the American College of Cardiology/American Heart Association Task Force on Practice Guidelines and the European Society of Cardiology Committee for Practice Guidelines. Circulation. 2003; 108: 1871-1909, doi: 10.1161/01.cir.0000091380.04100.84, indexed in Pubmed: 14557344.

13. Kirchhof P, Benussi S, Kotecha D, et al. 2016 ESC Guidelines for the management of atrial fibrillation developed in collaboration with EACTS: The Task Force for the management of atrial fibrillation of the European Society of Cardiology (ESC) Developed with the special contribution of the European Heart Rhythm Association (EHRA) of the ESC Endorsed by the European Stroke Organisation (ESO). Kardiol Pol. 2016; 74(12): 1359-1469, doi: 10.5603/KP.2016.0172, indexed in Pubmed: 28009037.

14. Page RL, Joglar JA, Caldwell MA, et al. 2015 ACC/AHA/HRS guideline for the management of adult patients with supraventricular tachycardia: Executive summary: A Report of the American College of Cardiology/American Heart Association Task Force on Clinical Practice Guidelines and the Heart Rhythm Society. Heart Rhythm. 2016; 13(4): e92-135, doi: 10.1016/j. hrthm.2015.09.018, indexed in Pubmed: 26409097.

15. Sharma AD, Klein GJ, Guiraudon GM, et al. Atrial fibrillation in patients with Wolff-Parkinson-White syndrome: incidence after surgical ablation of the accessory pathway. Circulation. 1985; 72(1): 161-169, doi: 10.1161/01.cir.72.1.161, indexed in Pubmed: 4006127. 
16. Waspe L, Brodman R, Kim S, et al. Susceptibility to atrial fibrillation and ventricular tachyarrhythmia in the Wolff-ParkinsonWhite syndrome: Role of the accessory pathway. Am Heart J. 1986; 112(6): 1141-1152, doi: 10.1016/0002-8703(86)90342-x, indexed in Pubmed: 3788760.

17. Chen PS, Pressley JC, Tang AS, et al. New observations on atrial fibrillation before and after surgical treatment in patients with the Wolff-Parkinson-White syndrome. J Am Coll Cardiol. 1992; 19(5): 974-981, doi: 10.1016/0735-1097(92)90281-q, indexed in Pubmed: 1552122.

18. Haissaguerre M, Fischer B, Labbé T, et al. Frequency of recurrent atrial fibrillation after catheter ablation of overt accessory pathways. Am J Cardiol. 1992; 69(5): 493-497, doi: 10.1016/00029149(92)90992-8, indexed in Pubmed: 1736613.

19. Dagres N, Clague JR, Lottkamp H, et al. Impact of radiofrequency catheter ablation of accessory pathways on the frequency of atrial fibrillation during long-term follow-up; high recurrence rate of atrial fibrillation in patients older than 50 years of age. Eur Heart J. 2001; 22(5): 423-427, doi: 10.1053/euhj.2000.2429, indexed in Pubmed: 11207084.

20. Miyamoto KJ, Tsuchihashi K, Uno K, et al. Studies on the prevalence of complicated atrial arrhythmias, flutter, and fibrillation in patients with reciprocating supraventricular tachycardia before and after successful catheter ablation. Pacing Clin Electrophysiol. 2001; 24(6): 969-978, doi: 10.1046/j.1460-9592.2001.00969.x, indexed in Pubmed: 11449594.

21. Kawabata M, Goya M, Takagi T, et al. The impact of B-type natriuretic peptide levels on the suppression of accompanying atrial fibrillation in Wolff-Parkinson-White syndrome patients after accessory pathway ablation. J Cardiol. 2016; 68(6): 485-491, doi: 10.1016/j.jjcc.2016.01.007, indexed in Pubmed: 26917195.

22. Orczykowski M, Walczak F, Derejko P, et al. Ventricular fibrillation risk factors in over one thousand patients with accessory pathways. Int J Cardiol. 2013; 167(2): 525-530, doi: 10.1016/j. ijcard.2012.01.076, indexed in Pubmed: 22357421.

23. Cosio FG, Anderson RH, Kuck KH, et al. Living Anatomy of the Atrioventricular Junctions. A Guide to Electrophysiologic Mapping : A Consensus Statement from the Cardiac Nomenclature Study Group, Working Group of Arrhythmias, European Society of Cardiology, and the Task Force on Cardiac Nomenclature from NASPE. Circulation. 1999; 100(5): e31-e37, doi: 10.1161/01. cir.100.5.e31.

24. Borregaard R, Lukac P, Gerdes C, et al. Radiofrequency ablation of accessory pathways in patients with the Wolff-ParkinsonWhite syndrome: the long-term mortality and risk of atrial fibrillation. Europace. 2015; 17(1): 117-122, doi: 10.1093/europace/ euu176, indexed in Pubmed: 25013013.

25. Sacher F, Wright M, Tedrow UB, et al. Wolff-Parkinson-White ablation after a prior failure: a 7-year multicentre experience. Europace. 2010; 12(6): 835-841, doi: 10.1093/europace/euq050, indexed in Pubmed: 20223787.

26. Kirchhof P, Lip GYH, Van Gelder IC, et al. Comprehensive risk reduction in patients with atrial fibrillation: emerging diagnostic and therapeutic options--a report from the 3rd Atrial Fibrillation Competence NETwork/European Heart Rhythm Association consensus conference. Europace. 2012; 14(1): 8-27, doi: 10.1093/europace/eur241, indexed in Pubmed: 21791573. 